Revista lus et Praxis, Año 20, No 2, 2014, pp. 473 - 494

ISSN 0717 - 2877

Universidad de Talca - Facultad de Ciencias Jurídicas y Sociales

"Prestaciones no contributivas de los trabajadores migrantes: un desafío pendiente en Latinoamérica"

Pablo Arellano Ortiz

Colaboración recibida el 16 de abril y aprobada el 20 de mayo de 2014

\title{
Prestaciones no contributivas de los trabajadores migrantes: un desafío pendiente en Latinoamérica*
}

\section{Pablo Arellano Ortiz}

De acuerdo a estimaciones de la Organización Internacional del Trabajo ${ }^{1}$ existen cerca de 214 millones de migrantes en el mundo. Esto significa que alrededor del 3 por ciento de la población mundial puede ser clasificada como migrante ${ }^{2}$. Cerca de la mitad de todos los migrantes son mujeres, y un significativo porcentaje de ellos son trabajadores migrantes, aunque existen otras categorías.

A nuestro entender toda persona tiene derecho a las prestaciones de seguridad social no importando si es migrante o nacional de un determinado país. Así, un residente o nacional de un país puede ser destinatario de diversas prestaciones para cubrir sus riesgos sociales. Para ello, los Estados establecen diversos mecanismos que permiten a través de sus prestaciones lograr entregar, en lo posible, una cobertura universal de la seguridad social. Para los efectos de este trabajo consideraremos a la seguridad social "en cuanto rama del derecho, como el conjunto integrado de principios, de normas e instituciones de ordenación estatal, destinadas a otorgar protección a las personas frente a las contingencias o estados de necesidad (riesgos sociales) a través de prestaciones (transferencias) en dinero o en especie, de financiamiento contributivo o no

\footnotetext{
* Este trabajo forma parte de los proyectos Fondecyt Iniciación № 11121677 "La coordinación de sistemas de seguridad social: Estudio comparado Unión Europea e Iberoamérica" y del Proyecto DID № 2012-44 Universidad Austral de Chile "Los mecanismos de coordinación de sistemas de seguridad social: Europa e Iberoamérica". El autor agradece a Francisca González Ortiz por su valiosa ayuda en la preparación de este trabajo.

** Profesor de Derecho del Trabajo y de Seguridad Social, Universidad Austral de Chile (Valdivia, Chile). Doctor en Derecho (Université Paris Ouest Nanterre La Defense, France); Master 2 Recherche Droit Social et Droit de la Santé (Université de Paris X Nanterre, France); Master 2 Recherche Droit Social (Université de Paris II Panthéon Assas, France). Correo electrónico: pablo.arellano@uach.cl.

1 HIROSE, Kenichi; NIKAC, Milos y TAMAGNo, Edward (2011): Social security for migrant workers. A rightbased approach (Budapest ILO, International Labour Organization, Decent Work Technical Support Team, and Country Office for Central and Eastern Europe), p. 2.

2 UNDP (2009): Overcoming Barriers: Human Mobility and Development (Human Development Report) (Basingstoke, Palgrave Macmillan), p. 9.
} 
contributivo, cuya administración puede ser pública y/o privada, debiendo el Estado asegurar un mínimo de protección a su población" ${ }^{\prime 3}$.

Sin embargo, la protección del trabajador migrante mediante la seguridad social no se encuentra exenta de dificultades. Esto debido a que las prestaciones de seguridad social se entregan generalmente en base a requisitos de calificación, que puede ser el cumplimiento de un número de cotizaciones, de un período de servicio o de residencia, entre otros. A esto debemos agregar que la afiliación al sistema nacional de seguridad social puede ser un requisito para la obtención de beneficios, como por ejemplo para la protección en caso de enfermedad o en caso de accidente de trabajo. Es decir, para obtener prestaciones se debe cumplir con ciertas condiciones, las que, en general, son cumplidas por el trabajador migrante en situación migratoria regular y que pertenece al sector formal ${ }^{4}$, ya que éste, al poseer una situación migratoria regular, se encuentra en principio afiliado al sistema nacional de seguridad social del país anfitrión.

Para poder entregar protección de seguridad social a los trabajadores migrantes se han desarrollado dos técnicas que permiten una cierta uniformización de legislaciones: la coordinación y la armonización. Cuando lo que se intenta es que los países involucrados adopten la misma norma jurídica se habla de armonización. En la coordinación, en cambio, esto no se produce pues cada país conserva su propio modelo social y su propia autonomía de legislación. Entendido así, resulta difícil que en materia de seguridad social se acuerde una armonización, ya que ello implicaría que los países perderían injerencia en su propia cultura de protección de riesgos sociales. Una misma ley de seguridad social aplicable a dos países es poco realista, pero que dos legislaciones puedan comunicarse y tengan puntos de conexión es mucho más viable. La coordinación entonces es la que permite esta comunicación ${ }^{5}$.

\footnotetext{
3 Cifuentes Lillo, Hugo, Arellano Ortiz, Pablo y Walker Errázuriz, Francisco (2013): Seguridad social, Parte General y Pensiones (Santiago, Librotecnia), p. 42. Para otras definiciones en la doctrina chilena ver: Humeres Noguer, Héctor (2005): Derecho del Trabajo y de la Seguridad Social, Tomo III, Derecho de la Seguridad Social, Decimoséptima edición ampliada y actualizada (Santiago, Editorial Jurídica de Chile; Novoa Fuenzalida, Patricio (1977): Derecho de la Seguridad Social (Santiago, Editorial Jurídica de Chile).

${ }^{4}$ Hirose, Kenichi; Nikac, Milos y TAmagno, Edward (2011): Social security for migrant workers. A rightbased approach (Budapest ILO, International Labour Organization, Decent Work Technical Support Team, and Country Office for Central and Eastern Europe), p. 2.

${ }^{5}$ Ver: Arellano Ortiz, Pablo (2013 a): "Social Security Protection for Migrants: A Need for Legal Intervention", en Blanpain, Roger; Arellano, Pablo; Olivier, Marius \& Vonk, Gisjbert (eds.), Bulletin of Comparative Labour Relations (Vol. 84), en: Social Security and Migrant Workers, Selected Studies of Cross-Border Social Security Mechanisms (Kluwer), pp. 31- 45; Arellano Ortiz, Pablo (2013 b): "Trabajadores migrantes y seguridad social: aproximación nacional e internacional a los mecanismos de protección que otorgan continuidad a sus prestaciones, II Parte", en: Revista Chilena de Derecho
} 
Para efectos de este trabajo se seguirá la definición de la OIT, la cual señala que: "La coordinación significa establecer mecanismos a través de los cuales los sistemas de seguridad social de diferentes países pueden trabajar juntos para lograr un conjunto de objetivos acordados -en particular para asegurar que los trabajadores migrantes y los miembros de sus familias tengan protección tan completa y continua como sea posible- mientras, al mismo tiempo, mantenga y respete las definiciones y reglas de cada sistema por separado. Coordinación no involucra remplazar las diferentes definiciones y reglas de cada sistema con definiciones y reglas comunes, lo que es usualmente referido como armonización" ${ }^{\prime \prime}$.

Los mecanismos que tiene la seguridad social para entregar continuidad a las prestaciones de seguridad social para las personas trabajadoras migrantes y sus familias, pueden ser unilaterales, bilaterales y multilaterales ${ }^{7}$. De ellas tan solo las bilaterales y multilaterales implican un cierto grado de coordinación, ya que las unilaterales son aquellas que adopta el Estado anfitrión o el Estado enviante de migrantes, con el fin de proteger a los migrantes y a sus familias en su territorio o a sus nacionales en el extranjero.

Sin embargo, la coordinación de sistemas de seguridad social, ya sea a través de medidas bilaterales o multilaterales, está principalmente destinada a los trabajadores migrantes en situación regular, o sea aquellos que pertenecen a la economía formal. Quedan entonces excluidos los trabajadores migrantes

de Trabajo y de la Seguridad Social, Departamento de Derecho de Trabajo y de la Seguridad Social, Facultad de Derecho, Universidad de Chile (Vol. 4, № 7), pp. 119-133.

${ }^{6}$ Hirose, Kenichi; Nikac, Milos y TAmagno, Edward (2011): Social security for migrant workers. A rightbased approach (Budapest ILO, International Labour Organization, Decent Work Technical Support Team, and Country Office for Central and Eastern Europe), p. 24.

7 Para una revisión de las tendencias mundiales sobre estos temas revisar: BLANPAIN, Roger; ARELLANO, Pablo; Olivier, Marius y Vonk Gisjbert (eds.) (2013): "Bulletin of Comparative Labour Relations" (Vol. 84), en: Social Security and Migrant Workers, Selected Studies of Cross-Border Social Security Mechanisms (Kluwer); HiROSE, Kenichi; Nikac, Milos y Tamagno, Edward (2011): Social security for migrant workers. A right-based approach (Budapest ILO, International Labour Organization, Decent Work Technical Support Team, and Country Office for Central and Eastern Europe); Rodríguez -PIÑEIRO Bravo FerRer, Miguel (2004): "La OIT y los trabajadores migrantes", en: Javillier, Jean-Claude y Gernigon, Bernard, Les normes internationales du travail: un patrimoine pourl'avenir. Mélanges en l'honneur de Nicolas Valticos (Geneva, Bureau International du Travail), p. 549; SABATES-WheELER, Rachel y KoetTL, Johannes (2010): "Protección social para los migrantes: los desafíos de la prestación en el contexto del cambio en los flujos migratorios", en: Revista Internacional de Seguridad Social (Vol. 63, 3-4/2010), p. 138; OIT (2010 b): International labour migration, A rights-based approach, International Labour Office (Geneva); OIT (2004): En busca de un compromiso equitativo para los trabajadores migrantes en la economía globalizada, Informe VI, Sexto punto del orden del día Conferencia Internacional del

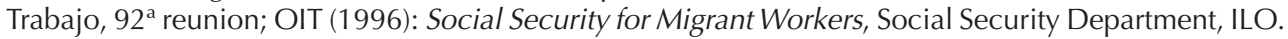


informales y todos aquellos que por diversos motivos no pueden participar de algún mecanismo de seguridad social ${ }^{8}$.

La coordinación como forma de entregar continuidad ha sido desarrollada principalmente a través de los reglamentos de coordinación de sistemas de seguridad social de los países de la Unión Europea, que regulan esta materia desde la época de las Comunidades Europeas hasta nuestros días. En dichos textos se incluyen disposiciones que permiten al migrante beneficiarse de prestaciones contributivas y no contributivas. En América Latina la posibilidad de un acuerdo regional que regule esta materia es bastante reciente. Así es como encontramos el acuerdo regional de coordinación, promovido por la Organización Iberoamericana de Seguridad Social, denominado Convenio Multilateral Iberoamericano de Seguridad Social, el cual tiene limitado su campo material de aplicación comprendiendo solo prestaciones periódicas de financiamiento contributivo ${ }^{9}$.

De una manera general se puede afirmar que la seguridad social en América Latina se basa en los mecanismos de protección tipo regímenes bismarkianos, es decir, seguros sociales basados en el trabajo dependiente ${ }^{10}$. Sin embargo, un porcentaje importante de la población cubierta realiza un trabajo dependiente a través de su participación en el mercado de trabajo formal y en virtud del cual contribuyen a la seguridad social. Pero la estructura del mercado de trabajo en la región latinoamericana es diferente a la europea y obliga a reconsiderar las vías de financiamiento tradicional de la seguridad social en esta región a través de cotizaciones, contribuciones o aportaciones, con el objeto de ampliar la cobertura para proteger a los marginados y a su vez lograr que el Estado aumente su participación financiera ${ }^{11}$. Dicho aumento se logra a través de los mecanismos de protección financiados por medio de impuestos, es decir prestaciones no contributivas.

\footnotetext{
8 Para un estudio más acabado de estos aspectos ver: Arellano Ortiz, Pablo (2013 d), "Características y desafíos de la migración para la seguridad social en la región latinoamericana", en: Revista Chilena de Derecho y Ciencia Política, Universidad Católica de Temuco (Vol. 4, № 1), pp. 15-42.

9 Para información sobre el texto del convenio y sobre su proceso de entrada en vigencia ver www. oiss.org. Ver además: Arellano Ortiz, Pablo y Briganti, Francesco (2012): "Europa y América Latina: encuentros y desencuentros en la búsqueda de la coordinación de los sistemas de seguridad social", en Programa de Estudios Europeos UDEC, Europa y América Latina. Dos caminos, ¿un destino común?, Programa de Estudios Europeos Universidad de Concepción (RIL Editores), pp. 125-155; JIMÉNEZ, Adolfo (2011): "Una experiencia inédita", en: Miradas al Exterior, Revista de Información Diplomática del Ministerio de Asuntos Exteriores y de Cooperación (abril-junio, №18), p. 19; SÁnchez-Rodas NaVArRo, Cristina (2011): "El Convenio Multilateral Iberoamericano de seguridad Social", en: IUSTEL (junio 2011).

10 OIT (1993): El seguro social y la protección social, Conferencia Internacional del Trabajo. 80ª reunión Memoria del director general (Parte I) (Ginebra).

11 LaCCHINI, Carlos y ZucCottı, Guillermo (2009): Importancia de la contributividad en el sistema de seguridad social. Elementos de análisis para la inclusión social en América Latina. Proyecto de seguridad social para organizaciones sindicales SSOS (Turín, CIF-OIT), p. 124.
} 
Para poder dimensionar el problema de la migración y la seguridad social en esta región es necesario tener en cuenta ciertos números: América Latina tiene una población total de 575.867.000 personas, de las cuales alrededor de 276.551.000 corresponden a la población activa total. En esta región se produjo una migración interna total de unos 25 millones de personas en el año 2005. Así vistas las cosas, América Latina es considerada la zona con mayor tasa de migración interna en el mundo, según cifras de la Comisión Económica para América Latina y el Caribe (Cepal), lo que deriva en que la vida laboral de muchos ciudadanos tiene lugar entre varios países de la región ${ }^{12}$. Además, el 78\% de la población migrante en la región Iberoamericana se encuentra en América Latina, un $20 \%$ en España y sólo el $2 \%$ en Portugal ${ }^{13}$. En términos cuantitativos el fenómeno migratorio interno en América Latina es muy importante y se ha llegado a señalar que muy pronto sobrepasará las cifras de migración interna de la región Europea.

Por otro lado, resulta necesario verificar si la seguridad social tradicional está entregando una completa cobertura en la región y un elemento a considerar es si las cotizaciones son efectuadas. En este sentido, se puede observar que el promedio de las cotizaciones en la región de América Latina para las y los trabajadores migrantes y nativos, es ligeramente superior al $50 \%$ de la población en ambos grupos. Sin embargo, la población migrante que trabaja en el sector formal tiene una cobertura inferior a la contribución de los nativos, en alrededor 10 puntos porcentuales. Esto podría deberse a que fueron en su mayoría empleados en pequeñas empresas, en las que finalmente hay una mayor irregularidad en el pago de las contribuciones. En el sector informal la situación es distinta, ya que quienes tienen una mayor cobertura son nativos, pero las diferencias son mucho más pequeñas, porque en ambos casos cerca de un tercio de los trabajadores cotiza. Otro signo de la vulnerabilidad de las personas migrantes, es la contribución de aquellos que tienen un contrato, la cual, también, es más de 10 puntos porcentuales más baja entre migrantes que entre nativos ${ }^{14}$. Estas cifras muestran claramente que el mecanismo de cotización tradicional no garantiza la cobertura universal en la región latinoamericana. Las y los trabajadores migrantes formales e informales tienen menor capacidad

\footnotetext{
12 JIMÉNEZ, Adolfo (2011): "Una experiencia inédita", en; Miradas al Exterior, Revista de Información Diplomática del Ministerio de Asuntos Exteriores y de Cooperación (abril-junio, № 18), p. 19.

13 Maguid, Alicia y Salinas, Viviana (2010): "Inserción laboral y acceso a mecanismos de seguridad social de los migrantes en Iberoamérica", en: Serie población y desarrollo (№ 96), Cepal, Naciones Unidas (EE.UU., CEPAL y ONU), p. 12.

14 Maguid, Alicia y Salinas, Viviana (2010): "Inserción laboral y acceso a mecanismos de seguridad social de los migrantes en Iberoamérica", en: Serie población y desarrollo (№ 96), Cepal, Naciones Unidas (EE.UU., CEPAL y ONU), p. 68.
} 
contributiva, lo que les impide tener acceso a prestaciones de seguridad social. Esto se puede apreciar claramente en la tabla $\mathrm{N}^{0} 1$.

TABLA 1

América Latina: Cotizaciones previsionales de migrantes y nativos 2005

\begin{tabular}{lcccccccc}
\hline \multirow{2}{*}{ País o región } & \multicolumn{2}{c}{ Cotizantes } & \multicolumn{2}{c}{$\begin{array}{c}\text { Cotizantes formales } \\
\text { total formales }\end{array}$} & $\begin{array}{c}\text { Cotizantes informales/ } \\
\text { total informales }\end{array}$ & \multicolumn{2}{c}{$\begin{array}{c}\text { Cotizantes entre los } \\
\text { que tienen contrato }\end{array}$} \\
\cline { 2 - 9 } & Nativos & Migrantes & Nativos & Migrantes & Nativos & Migrantes & Nativos & Migrantes \\
\hline América Latina & 56.4 & 52.5 & 69.0 & 58.2 & 33.9 & 36.8 & 81.5 & 67.5 \\
Argentina & 63.5 & 64.2 & 75.0 & 60.7 & 23.1 & 22.0 & 72.3 & 57.5 \\
Bolivia (Estado & 28.3 & 30.8 & 45.8 & 33.0 & 4.5 & - & 65.8 & 82.6 \\
Plurinacional de) & & & & & & & & \\
Brasil & 72.5 & 59.4 & 77.9 & 61.3 & 40.1 & 36.1 & 100.0 & 100.0 \\
Chile & 84.6 & 74.0 & 83.4 & 73.7 & 93.2 & 95.4 & 97.2 & 88.8 \\
Costa Rica & 81.7 & 74.5 & 90.8 & 86.6 & 41.5 & 31.7 & 80.2 & 68.2 \\
Ecuador & 46.7 & 73.2 & 62.6 & 75.5 & 15.0 & 64.0 & 80.3 & 80.8 \\
Guatemala & 48.9 & 10.9 & 66.3 & 13.2 & 7.6 & - & 83.0 & 10.6 \\
México & 62.6 & 44.9 & 76.3 & 54.2 & 20.2 & 0.5 & 84.3 & 55.2 \\
Nicaragua & 40.9 & 64.4 & 58.3 & 69.6 & 6.4 & - & 48.5 & - \\
República & 58.8 & 48.2 & 70.1 & 56.6 & 7.3 & - & 85.9 & 60.1 \\
Dominicana & & & & & & & & \\
Paraguay & 32.2 & 33.4 & 52.0 & 55.9 & 4.0 & 7.8 & 66.0 & 62.2 \\
\hline
\end{tabular}

Fuente: Maguid, Alicia y Salinas, Viviana (2010): "Inserción laboral y acceso a mecanismos de seguridad social de los migrantes en Iberoamérica", en: Serie población y desarrollo (№ 96), Cepal, Naciones Unidas (EE.UU., CEPAL y ONU), p. 68.

Teniendo en cuenta las características del fenómeno migratorio en América Latina y la importancia que éste tendrá en un mediano plazo, las características propias de los sistemas nacionales de seguridad social, que demuestran una baja capacidad contributiva de los trabajadores nativos y migrantes de la región, notamos que el trabajador migrante se encuentra en una situación desfavorecida frente a los migrantes en la región europea. Dicha región ofrece una cobertura de seguridad social basada también en los seguros bismarkianos, pero no posee los índices de informalidad que posee América Latina. De esta manera sostenemos que se hace imprescindible que se considere en los mecanismos a las prestaciones no contributivas dentro de los acuerdos regionales de coordinación de sistemas de seguridad social en la región latinoamericana. Para ello, una revisión de la normativa europea vigente sobre prestaciones no contributivas nos parece el paso previo para cualquier decisión debido a la fuerte influencia que dicha normativa ha tenido en la coordinación latinoamericana. 
Nuestro análisis se efectuará en tres tiempos, en los que intentaremos profundizar nuestro postulado viendo las deficiencias normativas de la protección del migrante en América Latina y los mecanismos existentes en la Unión Europea. Primero, a modo de una introducción al tema particular sobre las prestaciones en seguridad social, incluiremos un acápite que las explique y además muestre el desarrollo que estas han tenido en la búsqueda de la extensión de la seguridad social, evolución en la extensión que a nuestro parecer debe incluir al trabajador migrante. En segundo lugar se agregan dos apartados destinados a realizar una descripción normativa de los instrumentos de coordinación, por un lado del Convenio Multilateral Iberoamericano de Seguridad Social, y por otro, de las medidas contenidas en los reglamentos de coordinación europeos. En un tercer lugar consagraremos un espacio para, a propósito de las normas enunciadas, buscar los argumentos que permitan la incorporación de las prestaciones no contributivas a la regulación de nuestra región. Finalizaremos con algunas conclusiones sobre lo analizado.

\section{Prestaciones de seguridad social y su extensión: extensión de cobertura en expansión}

Nos parece necesario antes de avanzar más en detalle con el objeto de este estudio, y resaltar la importancia de las prestaciones no contributivas, especificar los alcances de ciertos conceptos, para ello intentaremos explicitar qué es lo que entendemos por seguridad social, por sus mecanismos protectores y además haremos una referencia al piso de protección social, promovido por la OIT para extender la cobertura a sectores excluidos de protección.

La seguridad social implica la adopción de todas las medidas relacionadas con las prestaciones, en efectivo o en especie, encaminadas a garantizar una protección en determinados casos, como por ejemplo: falta de ingresos laborales (o ingresos laborales insuficientes) debido a enfermedad, discapacidad, maternidad, accidentes de trabajo, desempleo, vejez o muerte de un miembro de la familia; falta de acceso o acceso a precios excesivos a la asistencia médica; apoyo familiar insuficiente, en particular para los hijos y adultos a cargo; pobreza generalizada y exclusión social.

Los sistemas de seguridad social pueden ser de carácter contributivo (seguro social) o de carácter no contributivo (asistencia social), aunque hoy en día en su mayoría poseen un carácter hibrido. Los regímenes contributivos y los no contributivos generalmente entregan prestaciones en especie, por ejemplo prestaciones médicas, y en dinero, el pago de pensiones y el pago de subsidios por incapacidad temporal ${ }^{15}$.

15 OIT (1984): Introducción a la seguridad social, Oficina Internacional del Trabajo (Ginebra). 
Las prestaciones contributivas poseen en general contenido económico, como por ejemplo las pensiones, cuya concesión está generalmente supeditada a una previa existencia de aportes o cotizaciones a la seguridad social. En general, para este tipo de prestaciones es necesario acreditar un período mínimo de cotización. La cuantía de la prestación se determina en función de las aportaciones, las cuales son en general efectuadas por la persona trabajadora y el empresario o empresaria, o sólo por la primera dependiendo de si se trata de un trabajo por cuenta ajena o independiente, durante el período que la legislación fije como necesario para la obtención de la prestación. Por otro lado, dentro de los sistemas contributivos puede darse una diferenciación entre los sistemas de administración pública y los sistemas de administración privada.

Las prestaciones no contributivas pueden ser asistenciales o no asistenciales. Las primeras son prestaciones económicas que se reconocen a aquellos ciudadanos que se encuentran en situación de necesidad y que carecen de recursos suficientes para su subsistencia y, generalmente, se somete al beneficiario potencial a una prueba de ingresos para determinar si califica para dicho beneficio, ya sea porque no ha cotizado nunca o, si lo ha hecho, el período de aporte es insuficiente para alcanzar las prestaciones del mecanismo contributivo. Por su parte, las prestaciones no contributivas y no asistenciales se asocian al cumplimiento de requisitos propios de regímenes para acceder a la prestación. Por ejemplo, en los países que tienen sistemas universales, que se financian con fondos públicos, la aportación al mecanismo se hace a través del pago de impuestos. El financiamiento de estas prestaciones proviene principalmente de impuestos de los ingresos generales y en algunos casos de otros impuestos específicos, dependiendo de cada legislación. Estas no tienen por su propia naturaleza una vinculación con el paso de la persona por el mercado formal de trabajo y otorgan prestaciones monetarias o en especie con diversos grados de uniformidad, conforme diferentes mecanismos de focalización.

No obstante, los regímenes no contributivos de seguridad pueden eventualmente entregar prestaciones a aquellos migrantes que no forman parte de la economía formal, e incluso a aquellos migrantes en situación migratoria irregular.

Las recientes evoluciones de la extensión de la seguridad social ${ }^{16}$ muestran un número importante de programas de protección de financiamiento no contributivo ${ }^{17}$, en esta región en particular, más que en otras regiones del mundo.

\footnotetext{
${ }^{16}$ Sobre las recientes evoluciones y sus problemáticas ver: ArelLANO OrTIZ, Pablo (2010): "Reflexiones sobre las evoluciones recientes en materia de protección de riesgos sociales a nivel internacional", en: Revista de Derecho Universidad Católica del Norte, (Vol.17, Nº 2), pp.167-183.

17 Al respecto revisar entre otros: OIT (2011 a): General Survey concerning social security instruments in light of the 2008. Declaration on Social Justice for a Fair Globalization. Third item on the agenda: Information and reports on the application of Conventions and Recommendations, Report of the Committee of Experts on the Application of Conventions and Recommendations (articles 19, 22 and
} 
Así, se encuentran programas como Bolsa Familia en Brasil, Progresa en México o Programa Puente en Chile. A este respecto, la OIT y las Naciones Unidas han promovido la implementación de un piso de protección social ${ }^{18}$ que tiene este tipo de programas, como un mecanismo que pueda ampliar la cobertura de la seguridad social para los trabajadores de la economía informal, y por ende, también, de los trabajadores migrantes.

El piso de protección social contenido en la Recomendación sobre los pisos de protección social, 2012 (núm. 202) ${ }^{19}$ puede ser un medio adecuado para darle efectividad al derecho a la seguridad social para los trabajadores migrantes y sus familias.

Dicha recomendación se basa en principios tales como la universalidad de la protección; la no discriminación; igualdad de género y capacidad de responder a las necesidades especiales y la inclusión social, en particular de las personas que trabajan en la economía informal. La estrategia de extensión propuesta por esta recomendación se enfoca en entregar cobertura a todas las personas, tanto en la economía formal como en la economía informal, apoyando el crecimiento del empleo formal y la reducción de la informalidad.

\section{RECUADRO N ${ }^{\circ} 1$}

De acuerdo al artículo 5 de la Recomendación los pisos de protección social deben comprender por lo menos las siguientes garantías básicas de seguridad social:

35 of the Constitution), Report III (Part 1B), International Labour Conference, 100th Session, 2011, published in march 2011; OIT (2011 b): Seguridad social para la justicia social y una globalización equitativa, Conferencia Internacional del Trabajo, $100^{a}$ reunión, Informe VI, Sexto punto del orden del día, Discusión recurrente sobre la protección social (seguridad social) en virtud de la Declaración de la OIT relativa a la justicia social para una globalización equitativa (Ginebra); OIT (2011 c): Piso de protección social para una globalización equitativa e inclusiva. Informe del Grupo consultivo presidido por Michelle Bachelet, Convocado por la OIT con colaboración de la OMS (Ginebra); OIT (2010 a): Extending social security to all, A guide through challenges and options, International Labour Office, Social Security Department, ILO (Geneva).

18 De esta manera se formó un grupo consultivo que elaboró un informe al respecto: OIT (2011 c): Piso de protección social para una globalización equitativa e inclusiva. Informe del Grupo consultivo presidido por Michelle Bachelet, Convocado por la OIT con colaboración de la OMS (Ginebra).

19 Sobre esta recomendación ver: Servals, Jean-Michel (2012): "The New ILO Recommendation on Social Security", en: E-Journal of International and Comparative Labour Studies (Vol. 1, Nºs. 3-4, october-december 2012), pp. 1-26; OIT (2011 c): Piso de protección social para una globalización equitativa e inclusiva. Informe del Grupo consultivo presidido por Michelle Bachelet, Convocado por la OIT con colaboración de la OMS (Ginebra); OIT (2010 a): Extending social security to all, A guide through challenges and options, International Labour Office, Social Security Department, ILO (Geneva). 
a) acceso a un conjunto de bienes y servicios definido a nivel nacional, que constituyen la atención de salud esencial, incluida la atención de la maternidad, que cumpla los criterios de disponibilidad, accesibilidad, aceptabilidad y calidad;

b) seguridad básica del ingreso para los niños, por lo menos equivalente a un nivel mínimo definido en el plano nacional, que asegure el acceso a la alimentación, la educación, los cuidados y cualesquiera otros bienes y servicios necesarios;

c) seguridad básica del ingreso, por lo menos equivalente a un nivel mínimo definido en el plano nacional, para las personas en edad activa que no puedan obtener ingresos suficientes, en particular en caso de enfermedad, desempleo, maternidad e invalidez, y;

d) seguridad básica del ingreso para las personas de edad, por lo menos equivalente a un nivel mínimo definido en el plano nacional.

Fuente: ILOb, 2011

En esta Recomendación se describe un marco de política para los Estados miembros para establecer y mantener los pisos de protección social nacionales que comprenden garantías básicas de seguridad social a fin de garantizar que todos los miembros de la sociedad gocen de al menos un nivel básico de seguridad social durante toda su vida. Estas garantías deben ser proporcionadas a todos los residentes y los niños, como se define en las leyes y reglamentos nacionales y con sujeción a las obligaciones internacionales existentes. Los migrantes y sus familias deben tener acceso a estas garantías básicas de seguridad social en el estado donde residen, así como en su país de origen. Un mecanismo de protección establecido sobre las bases de esta recomendación tendría un doble efecto, de lucha contra la informalidad y además de extensión de cobertura a los trabajadores migrantes.

La coordinación de sistemas de seguridad social y los diversos mecanismos que permiten entregar continuidad a las prestaciones de los trabajadores migrantes, que se enunciaron en la introducción, están destinados a los trabajadores formales. Sin embargo, la población migrante y la no cubierta por la seguridad social en América Latina puede ser protegida por mecanismos no contributivos. Esto debido a las características propias del mercado de trabajo, tales como la informalidad, y por los defectos de los sistemas de seguridad social, al no percibir suficientes cotizaciones.

Las prestaciones no contributivas aparecen entonces como una solución para extender la cobertura a los trabajadores migrantes. Pero el mecanismo de coordinación regional existente en América Latina no incluye dentro de su 
campo de acción estas prestaciones. Por su parte, el mecanismo más complejo de coordinación, el de la Unión Europea, sí posee una regulación para la coordinación de estas prestaciones. A continuación revisaremos ambos textos para apreciar sus diferencias para luego intentar establecer algunas conclusiones que permitan un avance sobre este tema.

\section{Campo de aplicación material del Convenio Multilateral Iberoamericano de Seguridad Social}

El Convenio Multilateral Iberoamericano de Seguridad Social posee características muy particulares ya que éste no es un acuerdo que tienda a generar un marco de integración regional como es el caso de los textos europeos. El origen de este convenio se encuentra en la $V$ Conferencia de Ministros y Máximos Responsables de Seguridad Social de los países de Iberoamérica, celebrada en Segovia (España), en el año 2005, y en los acuerdos surgidos de la XV y XVI Cumbres Iberoamericanas de Jefes de Estado y de Gobierno, que posteriormente Ilevaron a la aprobación del texto definitivo del Convenio Multilateral por parte de los Jefes de Estado y de Gobierno en la XVII Cumbre Iberoamericana de Santiago de Chile, en noviembre de 2007. En el año 2009, en las reuniones de Lisboa, se dio vía libre al contenido del Acuerdo de Aplicación. Durante todo el proceso de elaboración del Convenio la Secretaría General Iberoamericana (SEGIB) y la Organización Iberoamericana de Seguridad Social (OISS) han tenido un papel importante, siendo esta última la responsable de dar la asesoría técnica, a fin de cumplir con la solicitud de apoyo formulada por los Ministros y Máximos responsables de la Seguridad de Iberoamérica.

En el momento actual, el Convenio ha sido ratificado por Argentina, Brasil, Bolivia, Chile, Ecuador, El Salvador, España, Paraguay, Perú, Portugal, Uruguay y Venezuela. El Convenio ha entrado en vigor el 1 de mayo de 2011, que corresponde al primer día del tercer mes siguiente a la fecha en que se haya depositado el séptimo instrumento de ratificación, aceptación, aprobación o adhesión. Sin embargo, para su aplicación efectiva es necesario que los países hayan suscrito el Acuerdo de Aplicación. De esta manera el Convenio es plenamente efectivo en Bolivia, Brasil, Chile, Ecuador, El Salvador, España, Portugal, Paraguay y Uruguay. La plena eficacia en el resto de los países dependerá de la pronta suscripción del Acuerdo de Aplicación.

\begin{tabular}{|l|c|c|c|c|c|c|}
\hline $\begin{array}{l}\text { Países que lo } \\
\text { han firmado }\end{array}$ & $\begin{array}{c}\text { Fecha firma } \\
\text { convenio }\end{array}$ & $\begin{array}{c}\text { Fecha } \\
\text { ratificación }\end{array}$ & $\begin{array}{c}\text { Fecha deposi- } \\
\text { to instrumento } \\
\text { ratificación en } \\
\text { SEGIB-OISS }\end{array}$ & $\begin{array}{c}\text { Fecha suscrip- } \\
\text { ción acuerdo } \\
\text { aplicación }\end{array}$ & $\begin{array}{c}\text { Aplicación } \\
\text { efectiva del } \\
\text { convenio }\end{array}$ & Población \\
\hline Argentina & $10 / 11 / 2007$ & $09 / 06 / 2010$ & & & & \\
\hline Bolivia & $10 / 11 / 2007$ & $08 / 11 / 2010$ & $02 / 02 / 2011$ & $18 / 04 / 2011$ & $X$ & 10.426 .160 \\
\hline Brasil & $10 / 11 / 2007$ & $30 / 10 / 2009$ & $11 / 12 / 2009$ & $19 / 05 / 2011$ & $X$ & 190.755 .799 \\
\hline
\end{tabular}




\begin{tabular}{|l|c|c|c|c|c|r|}
\hline $\begin{array}{l}\text { Países que lo } \\
\text { han firmado }\end{array}$ & $\begin{array}{c}\text { Fecha firma } \\
\text { convenio }\end{array}$ & $\begin{array}{c}\text { Fecha } \\
\text { ratificación }\end{array}$ & $\begin{array}{c}\text { Fecha deposi- } \\
\text { to instrumento } \\
\text { ratificación en } \\
\text { SEGIB-OISS }\end{array}$ & $\begin{array}{c}\text { Fecha suscrip- } \\
\text { ción acuerdo } \\
\text { aplicación }\end{array}$ & $\begin{array}{c}\text { Aplicación } \\
\text { efectiva del } \\
\text { convenio }\end{array}$ & Población \\
\hline Chile & $10 / 11 / 2007$ & $18 / 11 / 2009$ & $30 / 11 / 2009$ & $01 / 09 / 2011$ & $X$ & 17.094 .275 \\
\hline Colombia & $26 / 11 / 2008$ & & & & & \\
\hline Costa Rica & $10 / 11 / 2007$ & & & & & \\
\hline Ecuador & $10 / 11 / 2007$ & $31 / 08 / 2009$ & $04 / 11 / 2009$ & $20 / 06 / 2011$ & $X$ & 14.306 .876 \\
\hline El Salvador & $10 / 11 / 2007$ & $29 / 05 / 2008$ & $04 / 09 / 2008$ & $17 / 11 / 2012$ & $X$ & 6.251 .495 \\
\hline España & $10 / 11 / 2007$ & $05 / 02 / 2010$ & $12 / 02 / 2010$ & $13 / 10 / 2010$ & $X$ & 47.021 .031 \\
\hline Paraguay & $10 / 11 / 2007$ & $15 / 12 / 2010$ & $09 / 02 / 2011$ & $28 / 10 / 2011$ & $X$ & 6.391 .000 \\
\hline Perú & $10 / 11 / 2007$ & $19 / 09 / 2013$ & & & & \\
\hline Portugal & $10 / 11 / 2007$ & $27 / 10 / 2010$ & $22 / 12 / 2010$ & $19 / 03 / 2013$ & & 11.400 .000 \\
\hline $\begin{array}{l}\text { República } \\
\text { Dominicana }\end{array}$ & $07 / 10 / 2011$ & & & & & \\
\hline Uruguay & $10 / 11 / 2007$ & $24 / 05 / 2011$ & $26 / 07 / 2011$ & $26 / 07 / 2011$ & $X$ & 3.356 .584 \\
\hline Venezuela & $10 / 11 / 2007$ & $16 / 02 / 2009$ & & & & \\
\hline
\end{tabular}

Fuente: www.oiss.org [visitado 14 de enero de 2014 a las 18:00 pm.]

Por su parte, la coordinación iberoamericana también centra su extensión material en los riesgos sociales concebidos en forma clásica. Pero, a diferencia de la coordinación europea, se toma un criterio pragmático y se limita los riesgos a cubrir. El Convenio Multilateral Iberoamericano cubre solamente las prestaciones monetarias por los riesgos de vejez, invalidez, supervivencia y por accidentes del trabajo y enfermedades profesionales. De esta manera el listado de riesgos clásicos que se incluyen en el Convenio № 102 sobre norma mínima de seguridad social de la OIT queda incompleto. Se excluyen por ende las prestaciones por enfermedad, por maternidad, las prestaciones familiares y la asistencia sanitaria. Así como también la asistencia sanitaria derivada de accidentes del trabajo o de una enfermedad profesional. Aunque se permite a los países incluir la atención sanitaria en forma bilateral o multilateral, de acuerdo al artículo 3.5 del Convenio.

El campo de aplicación material del Convenio está fijado en su artículo $3^{\circ}$, en donde se explicita que el Convenio se aplicará a la legislación relativa a las siguientes ramas: prestaciones económicas de invalidez, prestaciones económicas de vejez, prestaciones económicas de supervivencia y las prestaciones económicas de accidentes del trabajo y enfermedad profesional. El convenio consagra su Título II denominado "Disposiciones particulares para las distintas categorías de prestaciones" para detallar la forma cómo cada riesgo será coordinado. Así, el Capítulo 1 está dedicado a "Prestaciones de invalidez, vejez y supervivencia", el Capítulo 2 a la "Coordinación de regímenes y legislaciones basados en el ahorro y la capitalización", y finalmente el Capítulo 3 trata de 
las "Prestaciones de accidentes del trabajo y de enfermedad profesional". El contenido de las prestaciones es únicamente el pago periódico, es decir el pago de una pensión mensual por alguna de las contingencias señaladas. A mayor abundamiento, en el artículo 3.2 se explicita que el Convenio se aplica a todos los regímenes contributivos de seguridad social, ya sean generales o especiales.

En cuanto a las prestaciones objeto de este trabajo el artículo 3.4 del Convenio señala en forma clara y precisa que éste no se aplicará a los regímenes no contributivos, así como tampoco a la asistencia social, ni a los regímenes a favor de las víctimas de guerra o de sus consecuencias. Queda bastante clara la exclusión de todo tipo de prestaciones no contributivas cualquiera que sea el riesgo cubierto. Llama, además, la atención la última frase en relación a las prestaciones a favor de las víctimas de guerra o de sus consecuencias, en ella se puede notar la influencia del texto europeo en la redacción del iberoamericano. Esto debido a que en las regulaciones europeas dicha mención existe porque diversos países poseen prestaciones para dichos destinatarios.

\section{Prestaciones no contributivas en la coordinación europea}

Los mecanismos de coordinación de sistemas de seguridad social más complejos y completos son los europeos ${ }^{20}$. Ello no es producto del azar sino que de una larga evolución legislativa tendiente a proteger los derechos de los migrantes. Hay que recordar que uno de los principios básicos de las Comunidades Europeas y de la actual Unión Europea es la libre circulación, no solo de trabajadores, sino que también de personas ${ }^{21}$.

Los primeros reglamentos europeos sobre coordinación son los números 3 y 4 del año 1958. Sin embargo, al poco tiempo en 1963 la Comisión Europea empezó a trabajar con el fin de mejorar la legislación vigente, de esta manera surgen el Reglamento (CEE) No 1408/71 (adoptado oficialmente el 14 de junio de 1971) y su reglamento de aplicación, el Reglamento (CEE) 574/72 que sustituyeron la normativa anterior.

\footnotetext{
20 Para profundizar en la legislación europea sobre coordinación de sistemas de seguridad social revisar: Pennings, Frans (2012): European Social Security Law (Wolter Kluwer Law \& Business); Sánchez-RodAs Navarro, Cristina, (dir.) (2010): La Coordinación de los Sistemas de Seguridad Social. Los reglamentos 883/2004 y 987/2009 (Murcia, Laborum); CORNELISSEN, Rob (2010): "Les axes de réforme les príncipes généraux du reglement 883/2004", en: RDSS 2010, dossier spécial Le nouveau droit communautaire de la sécurité sociale, p. 5; JoRENS, Yves (2010): 50 years of social security coordination. Past -PresentFuture, Report of the conference celebrating the 50th Anniversary of the European Coordination of Social Security. European Commission, Directorate General for Employment, Social Affairs and Equal Opportunities, Publication Office of the European Union.

${ }^{21}$ Sobre este tema ver: Álvarez De Sotomayor, Lucia Dans (2012): "Libre circulación de trabajadores", en: Magdalena Nogueira Guastavino, Olga Fotinopoulou Basurko y José María Miranda Boto, Lecciones de Derecho Social de la Unión Europea (Valencia, Ediciones Tirant Lo Blanch), pp. 162-184.
} 
El Reglamento $N^{\circ}$ 1408/71 fue aplicado durante un largo lapso de tiempo sin que sufriera modificaciones sustanciales pero en los años noventa aparece la necesidad de reformarlo. La nueva regulación tomó tiempo en ser aprobada y así el nuevo reglamento llegó solamente en abril de 2004 (Reglamento 883/04), después de muchas discusiones difíciles y compromisos entre los Estados miembros. Además se adoptó el Reglamento 987/09 que proporciona medidas para la aplicación del Reglamento 883/2004. Los nuevos Reglamentos 883/04 y 987/09 luego de un largo período de preparación legislativa finalmente entran en vigor el 1 de mayo de $2010^{22}$.

Es dentro de las normas del Reglamento 883/2004 relativas a su campo de aplicación material donde encontramos la referencia a cuáles prestaciones se encuentran incluidas en la coordinación europea. Al respecto existe una amplia bibliografía sobre el campo de aplicación y de los problemas que estos presentan ${ }^{23}$.

En su artículo 3.1 el Reglamento 883/2004 se indica su ámbito de aplicación, el que abarca las siguientes ramas de la seguridad social: a) las prestaciones de enfermedad; b) las prestaciones de maternidad y de paternidad asimiladas; c) las prestaciones de invalidez; d) las prestaciones de vejez; e) las prestaciones de supervivencia; f) las prestaciones de accidentes de trabajo y de enfermedad profesional; g) los subsidios de defunción; h) las prestaciones de desempleo; i) las prestaciones de prejubilación; y j) las prestaciones familiares. Cabe hacer notar que este reglamento va más allá de las 9 ramas clásicas de la seguridad social, establecidas en el Convenio $N^{0} 102$ sobre norma mínima de seguridad social de la OIT, ya que se incluye, por ejemplo, a la paternidad y a las prestaciones de prejubilación.

Las legislaciones relativas a todos estos regímenes de seguridad social generales y especiales, contributivos y no contributivos, se encuentran incluidas en la coordinación, a menos que los países señalen lo contrario mediante una declaración que se incluye en el anexo XI del reglamento y, además, se excluye a los regímenes relativos a las obligaciones del empleador o del armador. De acuerdo al artículo $9^{\circ}$ del reglamento 883/2004 las declaraciones que realicen

\footnotetext{
22 Sánchez-Rodas Navarro, Cristina, (dir.) (2010): La Coordinación de los Sistemas de Seguridad Social. Los reglamentos 883/2004 y 987/2009 (Murcia, Laborum); CorneLISSEN, Rob (2010): "Les axes de réforme les príncipes généraux du reglement 883/2004", en: RDSS 2010, dossier spécial Le nouveau droit communautaire de la sécurité sociale.

23 Por ejemplo: Meléndez Morillo-Vélarde, Lourdes y Pérez Campos, Ana Isabel (2012): Materiales de derecho social comunitario, Teoría y práctica (Madrid, Editorial Tecnos); NavarRo Nieto, Federico, Rodríguez-Piñeiro Royo, Miguel C. y Gómez Muñoz, José Manuel (dir.) (2010): Manual de derecho social de la Unión Europea (Madrid, Editorial Tecnos), p. 133; SÁnchez-Rodas NAVArRo, Cristina (dir.) (2010): La Coordinación de los Sistemas de Seguridad Social. Los reglamentos 883/2004 y 987/2009 (Murcia, Laborum); CORNELISSEN, Rob (2010): "Les axes de réforme les príncipes généraux du reglement 883/2004", en: RDSS 2010, dossier spécial Le nouveau droit communautaire de la sécurité sociale.
} 
los estados deberán ser notificadas anualmente a la Comisión Europea y serán objeto de la publicidad necesaria. En dichas notificaciones se indica la fecha a partir de la cual el reglamento será aplicable a los regímenes especificados por los Estados miembros.

Hay que señalar que esta exclusión no significa que el Tribunal de Justicia de la Unión Europea quede vinculado por la notificación de su inclusión en el anexo correspondiente, ya que es este órgano el que está revestido de la facultad de intérprete supremo del derecho comunitario. Por lo que puede pronunciarse sobre la correcta o incorrecta incorporación en el anexo correspondiente ${ }^{24}$.

El reglamento, asimismo, establece ciertas precisiones en cuanto a las prestaciones no contributivas, así, en su artículo 3.3 se establece que el reglamento se aplica a las prestaciones especiales en metálico no contributivas previstas en el artículo 70, que a continuación detallaremos. Pero, además, señala, en su artículo 3.5, que el reglamento no se aplica: a) a la asistencia social y sanitaria, ni b) a las prestaciones respecto a las cuales un Estado miembro asuma la responsabilidad de los daños causados a las personas y prevea una compensación, tales como las concedidas a las víctimas de guerra y de acciones militares o de sus consecuencias; las víctimas de delitos, asesinato o actos terroristas; las víctimas de daños ocasionados por agentes del Estado miembro en el ejercicio de sus funciones, o las víctimas que se hayan visto perjudicadas por razones políticas o religiosas o debido a su origen.

De esta manera se puede observar que la coordinación europea incluye a las prestaciones no contributivas de manera general, incluyendo a aquellas denominadas prestaciones especiales en metálico no contributivas. Pero, por otro lado, deja fuera a las prestaciones de origen estatal, por ende no contributivas, que sean concedidas con ciertos motivos, y, además, excluye a la asistencia social y sanitaria. Cabe hacer notar que el concepto de seguridad social utilizado por la coordinación europea es restringido en cuanto a los mecanismos que se incluyen. La concepción clásica de seguridad social nace por la unión de los mecanismos de seguro social y de la asistencia social ${ }^{25}$, y el legislador europeo parece no incluir dentro de su seguridad social a las prestaciones de asistencia social.

Para tener una mejor apreciación de la manera como son incluidas las prestaciones no contributivas se analizarán dos materias específicas al respecto.

\footnotetext{
${ }^{24}$ SánChez-Rodas NAVARRo, Cristina (2010 a) : "La Coordinación de las prestaciones familiares en el nuevo Reglamento 883/2004", en: Sánchez-Rodas Navarro, Cristina (dir.) La Coordinación de los Sistemas de Seguridad Social. Los reglamentos 883/2004 y 987/2009 (Murcia, Laborum), p. 334.

25 Sobre esta discusión y sobre la evolución de la seguridad social ver: ArelLANO OrTIz, Pablo (2010): "Reflexiones sobre las evoluciones recientes en materia de protección de riesgos sociales a nivel internacional", en: Revista de Derecho Universidad Católica del Norte, (Vol.17, № 2), pp.167-183.
} 
Primero, se tratará la coordinación de las prestaciones familiares por ser este tipo de prestaciones en general en América Latina prestaciones no contributivas. En segundo lugar, trataremos el caso específico de las prestaciones especiales en metálico no contributivas.

\subsection{Coordinación de las prestaciones familiares}

Las prestaciones familiares ${ }^{26}$ se encuentran definidas en el Reglamento 883/2004 en su artículo 1.z) que señala que éstas son "todas las prestaciones en especie o en metálico destinadas a hacer frente a los gastos familiares, con exclusión de los anticipos de pensiones alimenticias y los subsidios especiales de natalidad y adopción mencionados en el anexo I". El contenido de su regulación dentro de la coordinación europea lo encontramos en los artículos 67 a 69 del Reglamento 883/2004 y en los artículos 58 a 61 del Reglamento $987 / 2009$. Cabe hacer notar que existe la posibilidad de excluir determinadas prestaciones del campo de aplicación del Reglamento 883/2004 incluyéndolas en el Anexo I, además cuando las prestaciones familiares son entregadas en forma de pensiones o de complementos de pensión, éstas se regirán por las reglas relativas a las pensiones de vejez y de supervivencia, de acuerdo al artículo 69.2 del Reglamento 883/2004. Existen a su vez disposiciones destinadas a regular la posibilidad de anti-cúmulo de prestaciones, artículo 68, y relativas al complemento diferencial, articulo 68.2.

El artículo 67 del Reglamento 883/2004 establece que "cualquier persona tendrá derecho a prestaciones familiares con arreglo a la legislación del Estado miembro competente, que serán extensivas a los miembros de su familia que residan en otro Estado miembro, como si residieran en el Estado miembro competente. No obstante, los titulares de pensiones tendrán derecho a prestaciones familiares con arreglo a la legislación del Estado miembro competente respecto de sus pensiones".

La coordinación de las prestaciones familiares se rige en derecho comunitario por ciertos principios. Estos poseen características particulares y que los hacen

${ }^{26}$ Revisar: Meléndez Morillo-VÉtarde, Lourdes y Pérez Campos, Ana Isabel (2012): Materiales de derecho social comunitario, Teoría y práctica (Madrid, Editorial Tecnos); Navarro Nieto, Federico, RodríguezPiñeiro Rovo, Miguel C. y Gómez Muñoz, José Manuel (dir.) (2010): Manual de derecho social de la Unión Europea (Madrid, Editorial Tecnos), p. 150. 
diferentes de los principios generales de la coordinación ${ }^{27}$. A continuación se realizará una breve revisión de estos principios ${ }^{28}$ :

\section{a.- Totalización sin prorrateo}

La totalización consiste en considerar todo el tiempo exigido como cotizado o como afiliado a un régimen en particular aun no habiéndolo cumplido para que se pueda tener derecho a la prestación. De esta manera, se consideran los tiempos exigidos como requisitos para obtener derecho a la prestación cumplidos en uno o más países distintos de aquel en que se solicita la prestación. En general, la técnica utilizada para reconocer estos períodos es mediante un prorrateo de lo cumplido en los otros países.

Sin embargo, para el caso de las prestaciones familiares este principio de la coordinación, la totalización, que generalmente se utiliza en regímenes contributivos sufre una modificación. Esto debido a que en general este tipo de prestaciones son sólo de cargo de un Estado. Entonces lo que hace la reglamentación europea es considerar como si se debiese hacer una totalización pero sin prorrateo. Ahora bien, se le denomina totalización sin prorrateo pero en realidad no existe totalización propiamente tal ya que ésta, en el caso de las prestaciones familiares, carece de sentido. Basta con que se acredite las condiciones de empleo o de residencia para en general tener derecho a las prestaciones familiares.

\section{b.- Inexportabilidad de prestaciones}

Para la reglamentación europea las prestaciones familiares nunca han sido objeto de exportabilidad, esto es, que las prestaciones sean entregadas fuera de los límites del país en el que se cumplieron los derechos para ella.

Ello es así porque si bien se puede determinar un Estado competente para que las entregue por hijos que se encuentren residiendo fuera de sus fronteras,

\footnotetext{
27 Los principios de la coordinación de los sistemas de seguridad social son: igualdad de trato, determinación de la legislación aplicable, mantenimiento de los derechos adquiridos, mantenimiento de los derechos en curso de adquisición, colaboración administrativa y técnica entre instituciones nacionales y reciprocidad. Para una explicación detallada de estos principios ver: ArELLANO OrTIZ, Pablo (2013 a): "Social Security Protection for Migrants: A Need for Legal Intervention", en Blanpain, Roger; Arellano, Pablo; Olivier, Marius \& Vonk, Gisjbert (eds.), Bulletin of Comparative Labour Relations (Vol. 84), en: Social Security and Migrant Workers, Selected Studies of Cross-Border Social Security Mechanisms (Kluwer), pp. 3-45; HIROSE, Kenichi; NIKAC, Milos y TAmagno, Edward (2011): Social security for migrant workers. A right-based approach (Budapest ILO, International Labour Organization, Decent Work Technical Support Team, and Country Office for Central and Eastern Europe).

28 SánChez-Rodas Navarro, Cristina (2010 a): "La Coordinación de las prestaciones familiares en el nuevo Reglamento 883/2004", en: Sánchez-Rodas Navarro, Cristina (dir.) La Coordinación de los Sistemas de Seguridad Social. Los reglamentos 883/2004 y 987/2009 (Murcia, Laborum), pp. 335-340.
} 
no se está frente a un supuesto de exportación en sentido estricto. Además, quien origina la prestación es el trabajador o el cesante, si fuese el caso, que se encuentra residiendo en el Estado competente.

En definitiva, no es una exportación propiamente tal que permita que las prestaciones familiares sean entregadas fuera de las fronteras del Estado competente. Sino que lo que hace el Reglamento 883/2004 es impedir que un Estado miembro de la Unión Europea pueda hacer depender la concesión o la cuantía de las prestaciones familiares al requisito de la residencia de los miembros de la familia del trabajador en el Estado que las otorga. Esto con el objeto de poder garantizar al trabajador migrante el ejercicio de su derecho a la libre circulación dentro del territorio europeo.

\section{c.- Estado competente}

Un tercer principio a analizar es determinar cuál es el Estado competente para la entrega de las prestaciones familiares. En este sentido se deben tener en cuenta las definiciones que el Reglamento 883/2004 establece en relación al Estado competente y a la Institución competente, a saber:

Artículo 1.s) "Estado miembro competente": el Estado miembro competente en el que se encuentra la institución competente;

Artículo 1.q) "institución competente":

i) la institución a la cual el interesado esté afiliado en el momento de la solicitud de prestaciones, $o$

ii) la institución de la cual el interesado tenga derecho a obtener prestaciones, o tendría derecho a ellas si él o uno o más miembros de su familia residieran en el Estado miembro donde se encuentra esta institución, o

iii) la institución designada por la autoridad competente del Estado miembro de que se trate, $o$

iv) si se trata de un régimen relativo a las obligaciones del empleador en relación con las prestaciones mencionadas en el apartado 1 del artículo 3, bien el empleador o el asegurador subrogado, bien, en su defecto, el organismo o la autoridad designada por la autoridad competente del Estado miembro de que se trate.

Entonces, en cuanto a la determinación del Estado competente, y siguiendo a Sánchez-Rodas ${ }^{29}$ es necesario hacer una distinción, de acuerdo a lo establecido en el artículo 67 del Reglamento 883/2004, entre una regla general y una excepción. La regla general consiste en que todas las personas protegidas

29 SÁnChez-Rodas NAVARro, Cristina (2010 a): "La Coordinación de las prestaciones familiares en el nuevo Reglamento 883/2004", en: Sánchez-Rodas Navarro, Cristina (dir.) La Coordinación de los Sistemas de Seguridad Social. Los reglamentos 883/2004 y 987/2009 (Murcia, Laborum), p. 339. 
por el Reglamento 883/2004 tienen derecho a las prestaciones familiares que les correspondan según la legislación del Estado que les resulte aplicable, ya sea al momento de la solicitud, afiliación, nacimiento del derecho, o por su relación de empleo. La excepción consiste en que las prestaciones familiares les serán abonadas a los titulares de pensiones por el Estado que corresponda que las pague.

\subsection{Prestaciones especiales en metálico no contributivas}

Estas prestaciones han constituido una categoría especial que ha dado lugar a una gran discusión al momento de su aplicación ${ }^{30}$. Se encuentran reguladas en el Capítulo 9 del Reglamento 883/2004 y más específicamente en el artículo 70.

Se comienza por señalar que las prestaciones de esta categoría son aquellas pertenecientes a la asistencia social de acuerdo al criterio del artículo 3.1. Para los efectos del reglamento se indica que se debe entender por "prestaciones especiales en metálico no contributivas" aquellas que:

“a) tienen por objeto proporcionar: i) cobertura adicional, sustitutoria o auxiliar de los riesgos cubiertos por las ramas de seguridad social mencionadas en el artículo 3.1, que garantice a las personas en cuestión unos ingresos mínimos de subsistencia respecto a la situación económica y social en el Estado miembro de que se trate, o ii) únicamente la protección específica de las personas con discapacidad, en estrecha vinculación con el contexto social de cada una de esas personas en el Estado miembro de que se trate, $y$

b) cuando la financiación proceda exclusivamente de la tributación obligatoria destinada a cubrir el gasto público general, y las condiciones de concesión y cálculo de las prestaciones, no dependan de ninguna contribución del beneficiario. No obstante, las prestaciones concedidas para completar una prestación contributiva no se considerarán prestaciones contributivas por este único motivo, y

c) figuren en el anexo $X$ del Reglamento".

Estas prestaciones deben ser facilitadas únicamente en el Estado miembro en el que las personas interesadas residan, y de conformidad con su legislación. Esas prestaciones deben ser facilitadas y sufragadas por la institución del lugar de residencia.

\footnotetext{
30 Sobre estas prestaciones ver: SÁnCHEZ-RodAS NAVARRO, Cristina (2010 b) "Libre circulación de personas y prestaciones en metálico no contributivas", en: Sánchez-Rodas Navarro, Cristina (dir.) La Coordinación de los Sistemas de Seguridad Social. Los reglamentos 883/2004 y 987/2009 (Murcia, Laborum), pp. 349-362; Meléndez Morillo-Vélarde, Lourdes y Pérez Campos, Ana Isabel (2012): Materiales de derecho social comunitario, Teoría y práctica (Madrid, Editorial Tecnos); NaVArRo Nieto, Federico, Rodríguez-Piñeiro Royo, Miguel C. y Gómez Muñoz, José Manuel (dir.) (2010): Manual de derecho social de la Unión Europea (Madrid, Editorial Tecnos), p. 151.
} 


\section{Textos normativos diferentes y contextos disímiles}

Sin lugar a dudas la decisión de excluir las prestaciones no contributivas del Convenio Multilateral Iberoamericano de Seguridad Social ha sido política. De hecho se destaca este carácter en el texto debido a que la región no posee la misma cohesión regional que la región europea y que la importancia de la adopción de este texto radica en la decisión política de los países miembros en abordar la protección de seguridad de los trabajadores migrantes de la región iberoamericana ${ }^{31}$.

Tanto en los sistemas europeos de seguridad como en los latinoamericanos uno de los principios básicos es el logro de la protección universal, tanto de todas las personas como también de todos los riesgos sociales. Existen en ambas regiones en los sistemas nacionales prestaciones contributivas y no contributivas. Sin embargo, los niveles de cobertura de la seguridad social en Europa son muy superiores. Ante esto se ha intentado buscar los mecanismos que permitan una mayor inclusión en la protección de todas las personas, incluidos los trabajadores migrantes. Así es como existe un fuerte movimiento que tiende a la extensión de la seguridad social a quienes por diversas razones se encuentran excluidos. Esto se ha llevado a cabo principalmente a través de prestaciones no contributivas ${ }^{32}$. Entonces, la discusión sobre la naturaleza de las prestaciones a incluir en la coordinación resulta de particular importancia en la región iberoamericana donde hoy en día se extiende la seguridad social a través de mecanismos no contributivos, como lo son el caso de Chile con su programa Chile Solidario y Bolivia con la Renta Dignidad ${ }^{33}$. Pero la importancia de estas prestaciones resulta ser mayor para América Latina que para los países europeos.

Como se describió a lo largo de este trabajo, en la Unión Europea existe un entramado complejo de normas que permiten al migrante poder gozar de las prestaciones de seguridad social cuando se desplaza dentro de dicha región.

\footnotetext{
${ }^{31}$ Jiménez, Adolfo (2011): "Una experiencia inédita", en: Miradas al Exterior, Revista de Información Diplomática del Ministerio de Asuntos Exteriores y de Cooperación (abril-junio, №18), p. 19.

32 Así se puede constatar en los informes de la OIT: OIT (2011 a): General Survey concerning social security instruments in light of the 2008. Declaration on Social Justice for a Fair Globalization. Third item on the agenda: Information and reports on the application of Conventions and Recommendations, Report of the Committee of Experts on the Application of Conventions and Recommendations (articles 19, 22 and 35 of the Constitution), Report III (Part 1B), International Labour Conference, 100th Session, 2011, published in march 2011; OIT (2011 b): Seguridad social para la justicia social y una globalización equitativa, Conferencia Internacional del Trabajo, 100ª reunión, Informe VI, Sexto punto del orden del día, Discusión recurrente sobre la protección social (seguridad social) en virtud de la Declaración de la OIT relativa a la justicia social para una globalización equitativa (Ginebra).

33 Arellano Ortiz, Pablo y Briganti, Francesco (2012): "Europa y América Latina: encuentros y desencuentros en la búsqueda de la coordinación de los sistemas de seguridad social", en Programa de Estudios Europeos UDEC, Europa y América Latina. Dos caminos, ¿un destino común?, Programa de Estudios Europeos Universidad de Concepción (RIL Editores), pp. 125-155.
} 
Los reglamentos europeos se han ocupado de proteger la continuidad de las prestaciones de seguridad social en forma completa incluyendo todos los riesgos sociales. Tal como se enunció, existen normas para coordinar específicamente prestaciones para ciertos riesgos sociales, que son generalmente entregadas a través de prestaciones no contributivas, como es el caso de las prestaciones familiares. El migrante latinoamericano, en cambio, se ve imposibilitado de recibir estas prestaciones no contributivas, lo que sin duda afecta el bienestar de los dependientes que se encuentren a su cargo.

Por otro lado, el fenómeno migratorio existe en ambas regiones, con un mayor acento en Latinoamérica, de lo cual deriva la necesidad que los migrantes sean protegidos por todos los mecanismos posibles. Vistas las características de la migración en la región latinoamericana, tales como el nivel de informalidad y la falta de capacidad contributiva, se hace necesario establecer una coordinación de las prestaciones no contributivas para los migrantes de la región.

Si se toma en cuenta el ejemplo chileno, la mayoría de los convenios bilaterales firmados por Chile establecen una coordinación para la vejez y en algunos casos para la salud ${ }^{34}$. Ninguno de ellos incluye prestaciones no contributivas.

Si bien existen numerosas diferencias entre el Convenio Multilateral Iberoamericano de Seguridad Social y los reglamentos europeos, la técnica legislativa existe para poder coordinar las prestaciones no contributivas. Una posible incorporación de estas medidas en la coordinación ya sea bilateral, pero sobre todo multilateral parece necesaria debido a las características de los migrantes y sus necesidades de protección, dentro del contexto de un mercado con las particularidades que posee el latinoamericano.

El Convenio Multilateral Iberoamericano de Seguridad Social constituye un paso importante en miras de la protección de seguridad social de los migrantes. Sin embargo, si lo enfrentamos a la regulación sobre coordinación europea encontramos fuertes diferencias, sobre todo por la inexistente preocupación en Europa por los trabajadores de la economía informal.

\section{Conclusiones}

De acuerdo a lo expresado a lo largo de este trabajo podemos resumir nuestra posición a favor de la incorporación de las prestaciones no contributivas a los mecanismos de coordinación en los siguientes argumentos:

\footnotetext{
${ }^{34}$ Sobre los convenio bilaterales de seguridad social firmados por Chile ver: ArELLANO ORTIZ, Pablo (2013 b): "Trabajadores migrantes y seguridad social: aproximación nacional e internacional a los mecanismos de protección que otorgan continuidad a sus prestaciones, II Parte", en: Revista Chilena de Derecho de Trabajo y de la Seguridad Social, Departamento de Derecho de Trabajo y de la Seguridad Social, Facultad de Derecho, Universidad de Chile (Vol. 4, № 7), pp. 119-133; CifUentes Lillo, Hugo (1998): "Tratados internacionales de seguridad social: convenios bilaterales", en: Estudios en Homenaje al profesor William Thayer (Santiago, Sociedad Chilena de Derecho del Trabajo y Seguridad Social), p. 21.
} 
a.- La exclusión de las prestaciones no contributivas del Convenio Multilateral Iberoamericano de Seguridad Social fue una decisión política y por tanto su inclusión implica, también, un nuevo acuerdo político.

b.- La extensión de la cobertura de la seguridad social en América Latina debe propender a la incorporación de prestaciones no contributivas, siguiendo la tendencia mundial al respecto y la Recomendación sobre los pisos de protección social, 2012 (núm. 202) de la OIT.

c.- Las normas de coordinación de prestaciones contributivas del Convenio Multilateral Iberoamericano de Seguridad Social fueron establecidas bajo una fuerte influencia de la reglamentación europea. Una revisión de éstas parece apropiada si se desea incorporar las prestaciones no contributivas para la región latinoamericana.

d.- La fuerte informalidad presente en la región latinoamericana indica que la debilidad de los sistemas de seguridad social debe ser remediada por las prestaciones de seguridad social. Si el migrante no tiene acceso a estas prestaciones la protección de su estado de necesidad se verá fuertemente afectada.

e.- La coordinación de sistemas de seguridad social en América Latina debe ser mirada como un proceso evolutivo, tal como lo fue en Europa. En este sentido, se debe ir mejorando y completando, siendo el paso a seguir la incorporación de las prestaciones no contributivas. 